\title{
Preface to Special Issue on Nanoscience and Catalysis
}

Over the past decade, to meet the ever increased demand on high-performance catalysts with excellent activity, selectivity and stability, the nanocatalysts and catalysis have been rigorously explored, resulting in a noticeable progress in new paradigm of nanoscience and nanotechnology for catalysis. Differing remarkably from conventional bulk catalysts, size shrinkage of active components to nanometer scale gives a rise to significantly increased catalytic activity, owing to the high surface-to-volume ratio of small particles as well as a large fraction of active atoms with dangling bonds exposed surfaces. In addition, other unique properties of nanomaterials such as surface- and strain-driven lattice distortion, variation in electronic state density and oxidation-induced charge redistribution could also benefit the catalytic performance.

Thanks to the rapidly advanced synthetic chemistry, nanomaterials with well-defined sizes, shapes, crystal facets, structures and compositions are readily obtainable, providing new means and opportunities to unlock catalytic activities of otherwise catalytic inactive materials via atomistic and nano-scale structural manipulations to produce catalysts with excellent activity to realise energetically challenging reactions, superior selectivity toward valuable products and long-term stability under tough reaction environments. To date, the development of nanocatalysts for thermal catalysis, electrocatalysis and photocatalysis in both China and Australia has achieved noticeable advances, which motivates us to put together a special issue dedicated to "Nano-Catalysis".

The issue was conceived as a collection of selected contributions by different researchers who are recognized as experts or rising stars in the field of nanomaterials-based catalyst synthesis, characterization and performance improvement. Although the final collection of papers represents only a short list, it is no doubt that these papers provide a unique overview of current research activities and directions. In the following, a brief description of papers included in this issue is provided to serve as an outline to encourage further reading.

The rational design and construction of catalysts with specific active sites is of great importance to achieve excellent performances for chemical transformations. Zhou et al synthesized $\mathrm{WO}_{x} / \mathrm{H}_{3} \mathrm{PO}_{4}$-activated montmorillonite (W/Mt-P) as catalysts for gas-phase dehydration of glycerol. The $12 \% \mathrm{~W} /$ Mt-P exhibited the highest catalytic performance with a glycerol conversion of $89.6 \%$ and an acrolein selectivity of $81.8 \%$.

Several contributions are related to the synthesis of electrocatalysts with complex composition as well as their applications in oxygen reduction reaction (ORR), oxygen evolution reaction (OER), hydrogen evolution reaction (HER), and so on. Cheng et al synthesized $\mathrm{Cu}$ /graphitic carbon nitride $(\mathrm{Cu} / \mathrm{g}-\mathrm{CN})$ as electrocatalysts for ORR, and they showed an impressive ORR catalytic activity. A $99 \mathrm{mV}$ positive shift of the onset potential and two times increased kinetic current density were clearly observed when compared with the pure g-CN. Jia et al demonstrated that the defective activated carbon (D-AC) supported 5\%wt $\mathrm{Pt}$ electrocatalysts surpassed the performance of commercial Pt/C with $20 \mathrm{wt} \% \mathrm{Pt}$ for the ORR in an alkaline solution. Wang et al demonstrated that a hydrotalcite-wrapped Co-B alloy was better for OER than that of a commercially available Ir/C catalyst. Sun et al reported that $\mathrm{MoS}_{2} / \mathrm{SNC}-900-12 \mathrm{~h}$ exhibited a low onset potential of $115 \mathrm{mV}$ with a low overpotential of $237 \mathrm{mV}$ at a current density of 10 $\mathrm{mA} \mathrm{cm}-2$ for HER. Furthermore, $\mathrm{MoS}_{2} / \mathrm{SNC}-900-12 \mathrm{~h}$ also had an excellent stability with only $\sim 2 \%$ decay at a current density of $10 \mathrm{~mA} \mathrm{~cm}^{-2}$ after 5000 test cycles. Feng et al synthesized porous bulk palladium samples via sodium chloride salt powder spacer incorporation and removal combined with dealloying with excellent electrocatalytic performance for ethanol oxidation. In addition, a review article authored by Kong et al described the 'big-picture' of recent advances made in the rational design of ORR electrocatalysts, including molecule-based, metal-oxide-based, metal-nanomaterial-based and two-dimensional electrocatalysts. A minireview article by Wang et al summarized the recent advances of amorphous catalysts for HER, OER and overall water splitting by electrolysis and the related fundamental chemical reactions.

Photocatalysis has become a highly important research field for solar-to-chemical energy conversion, and it also provides an 
effective means to organic transformations that cannot be carried out by conventional approaches. Yang et al demonstrated that amorphous ferric oxide loaded onto bismuth vanadate photoanode enabled efficient hole extraction and transfer for the existence of low-valence-state ferrous ion ( $\mathrm{Fe}^{\mathrm{II}}$ ), which resulted in a remarkably enhanced photocurrent $\left(2.52 \mathrm{~mA} \mathrm{~cm}^{-2}\right.$ at $1.23 \mathrm{~V}$ vs. RHE in $0.5 \mathrm{M}$ potassium phosphate buffer ( $\mathrm{pH} \sim 7.0)$ ). Wang et al showed that bismuth ferrite@ $\mathrm{TiO}_{2}$ core-shell nanocomposites exhibited excellent photocatalytic oxidation of methyl violet (MV) under both UV and visible light irradiations compared with pure $\mathrm{BiFeO}_{3}$ and $\mathrm{TiO}_{2}$. The enhancement of photocatalytic activities could be ascribed to the formation of $\mathrm{p}-\mathrm{n}$ junction of $\mathrm{BiFeO}_{3} / \mathrm{TiO}_{2}$ with improved charge separation efficiency as well as strong light absorption ability. Tang et al indicated that $\mathrm{Ag}_{2} \mathrm{O} / \mathrm{Ag}_{2} \mathrm{~S}_{2} \mathrm{O}_{7}$ heterostructure was a stable and efficient full-solar-spectrum photocatalyst. Jing et al showed that phosphate-oxygen (P-O) bridged $\mathrm{TiO}_{2} / \mathrm{g}-\mathrm{C}_{3} \mathrm{~N}_{4}$ nanocomposites had a threefold photocatalytic activity enhancement for degrading 2,4-DCP and $\mathrm{CO}_{2}$ reduction compared to bare g- $\mathrm{C}_{3} \mathrm{~N}_{4}$. The possible reason for the improved activity was that the photogenerated charge separation of $\mathrm{g}-\mathrm{C}_{3} \mathrm{~N}_{4}$ could be effectively enhanced when coupled with the $\mathrm{P}-\mathrm{O}$ bridged $\mathrm{TiO}_{2}$, resulting from the effective transfer of excited electrons from $\mathrm{g}_{-} \mathrm{C}_{3} \mathrm{~N}_{4}$ to $\mathrm{TiO}_{2}$. Zhao et al demonstrated that a highly efficient perovskite ( $\mathrm{La}_{0.6} \mathrm{Ca}_{0.4} \mathrm{Mn}_{0.6} \mathrm{Al}_{0.4} \mathrm{O}_{3}$ ) yielded a remarkable solar $\mathrm{H}_{2}$ production rate of $429 \mu \mathrm{mol} / \mathrm{g}$ from a two-step thermochemical $\mathrm{H}_{2} \mathrm{O}$ splitting process. Moreover, the performance of the optimized perovskite was found to be eight times higher than that of the benchmark catalyst $\mathrm{CeO}_{2}$ under the same experimental conditions.
Based on the above, the review article by Liu et al summarized recent progresses in the use of yolk shell structured nanoparticles as nanoreactors for various chemical transformations covering methane reforming, electrochemical conversion and photocatalysis. The state-of-the-art research and perspective in future development are also highlighted.

At last, we would like to thank all the authors who contributed to this thematic issue. Their contributions represent excellent examples of the current research trends in the field of nanocatalysts and catalysis. We also wish to thank the editorial staff of Chinese Journal of Catalysis for their help to organize this issue. We hope that the topics presented in this issue would inspire the readers to further explore the interesting science of nano-catalysis.

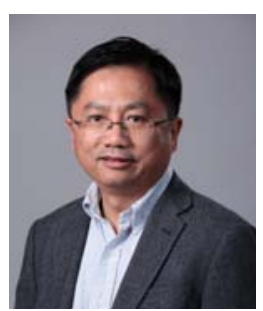

Professor Zhiyong Tang

CAS Key Laboratory of Nanosystem and Hierarchical Fabrication, CAS Center for Excellence in Nanoscience, National Center for Nanoscience and Technology, China

E-mail: zytang@nanoctr.cn

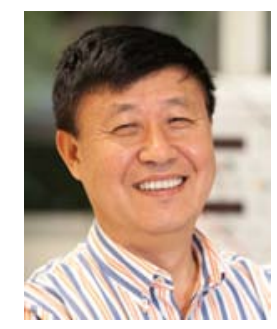

Professor Huijun Zhao

Centre for Clean Environment and Energy Gold Coast Campus, Griffith University, Australia E-mail: h.zhao@griffith.edu.au 